\title{
Medical Marijuana: Clearing Away the Smoke
}

\author{
Igor Grant* ${ }^{1}$, J. Hampton Atkinson ${ }^{1,2}$, Ben Gouaux ${ }^{1}$ and Barth Wilsey ${ }^{3}$ \\ ${ }^{1}$ Center for Medicinal Cannabis Research; University of California, San Diego; San Diego, CA, USA \\ ${ }_{2}^{2}$ Psychiatry Service; VA San Diego Healthcare System; San Diego, CA, USA \\ ${ }^{3}$ Department of Physical Medicine and Rehabilitation; University of California, Davis; Sacramento, CA, USA
}

\begin{abstract}
Recent advances in understanding of the mode of action of tetrahydrocannabinol and related cannabinoid ingredients of marijuana, plus the accumulating anecdotal reports on potential medical benefits have spurred increasing research into possible medicinal uses of cannabis. Recent clinical trials with smoked and vaporized marijuana, as well as other botanical extracts indicate the likelihood that the cannabinoids can be useful in the management of neuropathic pain, spasticity due to multiple sclerosis, and possibly other indications. As with all medications, benefits and risks need to be weighed in recommending cannabis to patients. We present an algorithm that may be useful to physicians in determining whether cannabis might be recommended as a treatment in jurisdictions where such use is permitted.
\end{abstract}

Keywords: Cannabis, chronic pain, pain.

\section{INTRODUCTION}

In this article we review evidence that cannabis may be useful as medicine. We discuss potential indications for its use and provide an algorithm to guide medicinal cannabis recommendations.

The reasons for a revival of interest in medicinal cannabis are multiple, and beyond the scope of this review, but include increasing anecdotal and clinical study reports of potential benefit, advances in understanding of the endocannabinoid signaling system upon which cannabis acts, as well as growing public acceptance that cannabis should be available as a medicine if a physician recommends it.

\section{BRIEF REVIEW OF PAST CLINICAL STUDIES ON MEDICINAL CANNABINOIDS}

As recently as a decade ago a review of the world literature on the status of the efficacy and safety of cannabinoids for pain and spasticity revealed that only nine randomized studies of acceptable quality had been conducted [1]. All of these were single dose studies comparing oral synthetic THC (or cannabinoid analogs or congeners) to codeine or placebo. Two were " $\mathrm{N}$ of 1 " randomized trials and two were of very small samples of acute post-operative pain. The remaining trials primarily addressed chronic cancer-related pain. Taken as a group it appeared that oral cannabinoids (e.g., THC $10 \mathrm{mg}$ ) outperformed placebo and were analgesically equivalent to codeine 60mg; higher doses (THC 20mg) were comparable to codeine $120 \mathrm{mg}$, but had a much higher incidence of adverse effects, particularly sedation [2]. Authoritative reviews judged cannabinoids as being unlikely to have a role in acute pain management, but suggested there was enough

*Address correspondence to this author at the Department of Psychiatry, School of Medicine; University of California San Diego, 9500 Gilman Drive; La Jolla, CA, 92093-0680; Tel: (858) 534-3652;

Fax: (858) 534-7723; E-mail: igrant@ucsd.edu evidence for efficacy in chronic neuropathic pain and muscle spasticity to warrant further research [1].

\section{RECENT STUDIES ON MEDICINAL CANNABIS}

In the past decade, the scope and rigor of research has increased dramatically. This research has employed cannabis, cannabis-based extracts, and synthetic cannabinoids delivered by smoking, vaporization, oral, and sublingual or mucosal routes.

\section{Studies on Smoked Cannabis}

Smoking cannabis provides rapid and efficient delivery of THC to brain. THC can be detected immediately in plasma after the first puff of a cigarette; peak concentrations occur within 10 minutes, then decrease to approximately $60 \%$ of peak by 15 minutes and $20 \%$ of peak by 30 minutes, but there can be wide inter-individual variation in concentrations achieved [3]. Rapid onset and predictable decay means that self-titration of dosing is attainable.

\section{Chronic Pain}

A series of randomized clinical trials at the University of California Center for Medicinal Cannabis Research (CMCR) investigated the short-term efficacy of smoked cannabis for neuropathic pain. Sponsored by the State of California Medical Marijuana Research Act of 1999, and conducted under the auspices of the Department of Health and Human Services, the National Institute on Drug Abuse, and the Food and Drug Administration, this research allocated participants to smoke cannabis cigarettes containing from $1 \%$ to $8 \%$ THC by weight (4 to $32 \mathrm{mg}$ THC) or to placebo cannabis cigarettes from which THC had been extracted. The total daily dose of THC ranged from $4 \mathrm{mg}$ to $128 \mathrm{mg}$. Two trials enrolled patients with painful HIV peripheral neuropathy [4, $5]$; one consisted of mixed neuropathic pain due to peripheral or central dysfunction of the nervous system (i.e., com- 
plex regional pain syndrome, peripheral neuropathy, and traumatic focal nerve or spinal cord injury) [6]. Patients were allowed to continue their usual regimen of analgesics. Results consistently indicated that cannabis significantly reduced pain intensity, with patients reporting 34\%-40\% decrease on cannabis compared to $17-20 \%$ on placebo. Moreover a significantly greater proportion of individuals reported at least $30 \%$ reduction in pain on cannabis (46\%$52 \%)$ compared to placebo (18\%-24\%) [4-6], which is relevant since $30 \%$ decrease in pain intensity is generally associated with reports of improved life quality [7]. The number needed-to-treat to achieve a $30 \%$ reduction in pain intensity was 3.5-4.5, a range achieved by standard non-opioid analgesics (i.e., noradrenergic antidepressants and anticonvulsants). Interestingly "medium" dose cannabis cigarettes (3.5\% THC) were as effective as higher dose (7\% THC) [6]. In this same vein, a fourth trial employing an experimental model of neuropathic pain (intradermal injection of capsaicin) in healthy volunteers suggested that there may be a "therapeutic window" or optimal dose for smoked cannabis: low dose cigarettes (2\% THC) had no analgesic effect, high dose (8\%) was associated with reports of significant pain increase, and medium dose cannabis cigarettes (4\% THC) provided significant analgesia [8]. Separately, another recent placebo-controlled, cross-over study of neuropathic pain due to surgery or injury examined the effect of $25 \mathrm{mg}$ doses of smoked cannabis at various potencies $(2.5 \%, 6 \%$, and $9.4 \%$ THC by weight), administered three times daily for 14 days [9]. Results suggested that although lower potency dosing was ineffective, 9.4\% THC produced modest but significant analgesic effects compared to placebo, in a sample selected for failure to respond to conventional therapy.

\section{Studies of Oral Preparations.}

Oral preparations are available as synthetic THC (dronabinol, Marinol ${ }^{\mathrm{R}}$ ) and a synthetic analog of THC (nabilone, Cesamet $^{\mathrm{R}}$ ). Absorption from the gut is slower and exhibits a delayed peak plasma concentration compared to smoking with bioavailability ranging from about $5-20 \%$ of dose; peak concentrations occur 1-6 hours after ingestion, with a magnitude approximately $10 \%$ of that achieved with smoking [3].

\section{Chronic Pain}

Most research using oral preparations has targeted neuropathic pain and spasticity associated with multiple sclerosis (MS). These randomized trials suggest that dronabinol (up to $25 \mathrm{mg}$ daily) significantly reduces pain compared to placebo (50\% "improved" on dronabinol compared to $30 \%$ on placebo, $\mathrm{p}<.05$ ) [10], with a number-needed-to-treat for $50 \%$ pain reduction of 3.5, which is in the range of efficacy observed for standard non-opioids [11]. Effects on spasticity are mixed: there may be no observable change in examinerrated muscle tone, but patients report significant relief [10].

There is less research with nabilone, although one threeweek randomized crossover trial reported that nabilone $2 \mathrm{mg}$ provided modest analgesia, comparable to dihydrocodeine 240mg daily in neuropathic pain [12].

\section{Nausea-Emesis and Appetite Stimulation}

Although serotonin receptor (5 HT3) antagonists (e.g., ondansetron, Zofran ${ }^{\mathrm{R}}$ ) and Substance P/neurokinin-1 (NK-1) receptor antagonists (e.g., aprepitant, Emend ${ }^{\mathrm{R}}$ ) are the mainstays for treatment, dronabinol and nabilone are also FDAapproved for control of acute and delayed nausea and emesis due to cancer chemotherapy. Meta-analyses indicate these cannabinoids are equivalent to or more effective than metoclopraminde and neuroleptics, but their side effect profile is less favorable in terms of sedation, dizziness, dysphoria, hypotension, and anxiety [13, 14]. There are no head-to-head comparisons of cannabinoids with serotonin 5 HT3 receptor or Substance P/NK-1 receptor antagonists.

Anorexia, early satiety, weight loss and cachexia are prevalent in late stage cancer and advanced HIV disease, but there are few effective treatments. Trials in AIDS patients with clinically significant weight loss indicated that dronabinol 5mg daily significantly outperformed placebo in terms of short term appetite enhancement (38\% vs. $8 \%$ at 6 weeks), and that these effects persisted for up to 12 months [15, 16], but were not accompanied by significant differences in weight gain, perhaps because of disease-associated energy wasting. The major practical limitations are the accompanying psychoactive side effects, and the problems of oral administration (eg, delayed onset of action, variable absorption, extended duration of effects).

\section{Studies on Cannabis-based Extracts}

Outside the US, extracts of whole plant are licensed and available in capsules (Cannador ${ }^{\mathrm{R}}$ ), with the main constituents being THC and the non-psychoactive plant cannabinoid, cannabidiol, in a ratio of 2:1. Rectal suppositories are also used to deliver THC hemisuccinate. Several small to medium-sized, randomized, controlled trials in MS suggest improvements in pain and perceived spasticity at daily doses of THC ranging from $7.5 \mathrm{mg}$ to $27.5 \mathrm{mg}[10,17,18]$. In some trials [19] but not others [10, 20] observer-assessed spasticity also improved.

\section{Studies with Alternative Delivery Systems}

The hazards of smoking and the pharmacokinetic limitations of ingestion of cannabinoids has led to a search for alternative systems of administration. One alternative is devices which vaporize cannabis leaves by heating the plant product to below the temperature of combustion (175-225 degrees C), permitting inhalation of volatilized gases minus hazardous pyrroles produced by burning. Preliminary work using plant material with a range of THC content (e.g., 1-7\% THC) suggests that there is rapid onset, with peak concentrations and six-hour area under the plasma concentration curves comparable to those achieved by smoking [21]. Vaporization is not a perfect solution since carbon monoxide is formed, but levels are significantly lower than with smoking [21]. Clinical trials are currently in progress at the CMCR assessing the efficacy of vaporized cannabis as an analgesic in chronic neuropathic pain.

Sublingual delivery systems of whole cannabis plant extract, which employ metered spray devices to deliver measured doses of THC (2.7mg) and cannabidiol (2.5mg), are undergoing Phase IIb/III trials in the US, and are licensed elsewhere for cancer pain and multiple sclerosis-associated neuropathic pain and spasticity (nabiximols, Sative ${ }^{\mathrm{R}}$ ). The apparent advantages of such systems are known cannabinoid 
concentrations, predetermined dosing aliquots, and time-out systems which may help prevent overuse. Some placebocontrolled trials suggest significant analgesia in neuropathic pain due to multiple sclerosis [22] and mixed neuropathy (e.g., post-herpetic, traumatic, vascular neuropathies, [23] but others do not [20]. Other controlled trials suggest efficacy for cancer-related pain inadequately responsive to opioid analgesia [24]. Responders participating in the open label extension phases of controlled trials appear to maintain analgesia on one-year follow-up [23].

In regard to spasticity in multiple sclerosis, a recent meta-analysis combining three trials with nabiximols in over 600 patients noted that mean intensity of patient rated spasticity was significantly reduced compared to placebo [20, 25, 26 , and that the proportion of "responders" (30\% reduction) was also significantly greater, with about $37 \%$ on the cannabinoid compared to $26 \%$ on placebo experiencing relief. Those reporting relief of spasticity seemed to maintain their gains over one year follow-up [27]. As with other studies noted above, observer-rated spasticity is often not reduced [20, 25, 28]; however, a recent CMCR study did find a significant reduction in observed spasticity among those administered active smoked marijuana vs. placebo marijuana [58].

\section{PRESERVATION OF MASKING IN CLINICAL TRIALS}

Because of the acute psychoactive effects of the experimental agent there is understandable concern that blinding cannot be preserved in placebo-controlled clinical trials of cannabinoids, particularly with cross-over designs. Few studies assess masking, but two cross-over trials tested maintenance of the blind by asking participants to "guess" assignment at different points of the study. Results suggest that participants, whether they are naïve or experienced cannabis users, are in the first week of a trial no more likely than by chance to guess assignment [5, 9]. With continued exposures rates of correct guesses exceed $75 \%$, but exceed chance only in a high potency arm (9\%) [9]. In another study correct guessing was related to two factors: whether the subject received placebo or cannabis first; and when during the study the participant guessed assignment [5]. Among individuals randomized to receive placebo first, guessing was no better than chance through the end of the first treatment week, whereas the majority of those randomized to receive cannabis first correctly guessed their treatment assignment at all time points. Furthermore, by the conclusion of the study, when all subjects had been given the opportunity to compare the cannabis placebo and treatments, even those randomized to receive placebo first correctly guessed their treatment assignment [5]. This raised the possibility that some of the pain reduction was placebo driven. Secondary analyses to assess whether correct treatment guessing influenced treatment responses showed that in the placebo group during the first treatment week, when guessing was no better than chance, cannabis still provided pain relief superior to that of placebo. This finding suggests that although placebo effects were present, treatment effects were independent [5].

\section{RISKS AND MANAGEMENT OF MEDICINAL USE OF CANNABINOIDS}

Acutely and over the longer term cannabis may have unwanted systemic and psychoactive adverse effects that must be taken into consideration in chronic pain populations, who have high rates of co-occurring medical illness (eg, cardiovascular disease) and co-morbid psychiatric and substance use disorders. In general these effects are dose-related, are of mild to moderate severity, appear to decline over time, and are reported less frequently in experienced than in naïve users. Reviews suggest the most frequent side effects are dizziness or lightheadedness (30\%-60\%), dry mouth (10\%-25\%), fatigue (5\%-40\%), muscle weakness (10\%-25\%), myalgia (25\%), and palpitations (20\%) [17]. Cough and throat irritation are reported in trials of smoked cannabis [9]. Tachycardia and postural hypotension are infrequent but caution is warranted in patients with cardiovascular disease, and possibly younger adults who intend to embark on very vigorous physical activity. At higher doses, sedation and ataxia with loss of balance are frequent. Participants in some but not all studies report euphoria: the relative absence of psychoactive effect has been attributed to the observation that plasma concentrations obtained in clinical trials are often $<25 \%$ of those achieved by "recreational” users (eg, $25 \mathrm{ng} / \mathrm{ml}$ vs $>100 \mathrm{ng} / \mathrm{ml}$ ) [9]. After repeated smoked or oral marijuana doses, tolerance is rapidly acquired (in two to 12 days) to many of its adverse effects, e.g., cardiovascular, autonomic, and many subjective and cognitive effects [29]. After exposure is stopped, tolerance is lost with similar rapidity.

There is little systematic data on timeline to tolerance of either adverse or therapeutic effects, like analgesia. Concerns have long been voiced that rapid tolerance to adverse effects might portend tolerance to beneficial effects [29]. Data from studies using oral sprays of cannabinoids or dronabinol in multiple sclerosis report that individuals can reduce the incidence and severity of adverse effects by downward selftitration without loss of analgesia [17]. Other studies in this population note that overall the incidence and severity of adverse effects diminishes over time without evidence of tolerance to analgesic effects [20, 22]. Yet it is rare that clinical trials of cannabinoids extend follow-up beyond 12 weeks, leaving questions on maintenance of gains or need for dose escalation unanswered [10, 26]. One study with 12month follow-up concluded there may be sustained analgesia for pain associated with multiple sclerosis, where about 30\% of cannabinoid-treated participants report continued "improvement" at 12 months compared to about $15 \%$ on placebo [30] on doses conservatively limited to a maximum of $25 \mathrm{mg}$ THC daily. This suggests that pain relief may be sustained without dose increases. But the study design was not intended to determine the proportion of patients who experienced diminution of effect, or whether dose escalation, even within the set boundary, was needed for maintenance of efficacy.

There are risks to be considered in assessing the potential of cannabinoid therapeutics. Cannabis, like other analgesics, can be associated with dependence and a withdrawal syndrome, occurring in a dose-dependent fashion [29]. Under controlled conditions in healthy, experienced users of marijuana, withdrawal from a "low" daily dose (ie, oral THC 10 mg every 3-4 hours for 5-21 days) commences within 12 hours, is diminished by 24 hours, and is complete in 48 to 72 hours [29]. Other short term experiments with oral THC (20 to 30mg four times daily) and smoked cannabis (1\% and 3\% THC cigarettes four times daily) reveal an abstinence syn- 
drome characterized by anxiety, irritability-restlessness, insomnia, stomach pain and decreased appetite [31, 32], with mood effects more prominent at the higher dosages. In research specifically designed to establish the time line of abstinence among regular heavy users (4 cigarettes daily), symptoms peak at 2 to 3 days, and persist for up to 2 weeks, although sleep disturbance may continue for up to 6 weeks [33]. In light of abstinence effects, standard practice in clinical trials administering a maximum of $25 \mathrm{mg}$ THC daily is to use a tapering scheme to conclude therapy, with a $20 \%$ per day dose reduction [30]. Patients discontinuing higher dose cannabinoids for analgesia might warrant a longer tapering regimen, but this has not been studied.

Fatal overdose with cannabis alone has not been reported. In terms of acute drug interactions, additive effects of cannabis, anticholinergics, and CNS depressants should expected (e.g., increased sedation, dizziness, dry mouth, confusion). Cannabinoids are metabolized by several enzyme systems, including Cytochrome P450 (CYP 2C9, CYP 3A4) and can induce or inhibit CYP 3A4, but there is little evidence of important drug-drug interactions based on CYP 450 systems. Smoking itself (e.g., cannabis or tobacco) induces CYP 1A2, and may increase clearance of some antipsychotics (e.g., olanzapine, clozapine) and antidepressants (e.g., some tricyclics, mirtazepine) [34, 35]. Overall then, the acute medical risks of THC as used in clinical trials are rather low.

There can be adverse psychiatric side effects. THC intoxication and euphoria can be disturbing, particularly to elderly patients. Anxiety and panic attacks occur, as do frank psychotic reactions (principally paranoia), as well as socalled "paradoxical" effects of dysphoria, dejection, and depressed mood [36, 37]. Although unlikely to be a factor in the application of cannabinoids for pain, there is concern that early adolescent use of cannabis may heighten later risk of psychosis [36, 38], and evidence that genetic variation (single nucleotide polymorphisms) heightens vulnerability [39].

Acute cannabinoid intoxication adversely impacts processing speed, attention, learning and recall, perception of time and velocity, reaction time and psychomotor abilities in a dose-dependent fashion [40]. Formal neuropsychological testing in clinical trials reveals mild impairment at usual analgesic doses [6, 23]. While cannabis can acutely impair skills required to drive motor vehicles in a dose-related fashion, epidemiological data are inconclusive with regard to the association of traffic accidents and use of cannabis [41]. There is speculation that cannabis use is associated with increased awareness of impairment (e.g., altered perception of time and speed), which results in compensatory behavioral strategies. What is clearer from experimental and epidemiologic data is that driving under the influence of both alcohol and cannabis in combination confers greater risk of accidents than the risk of either drug alone [41].

The longer-term health risks of cannabis are unclear, and the evidence is based on non-medical use [42]. Long-term use of inhaled cannabis may be associated with dependence and increased respiratory symptoms; but some epidemiological studies have not found more lung disease in long-term users, once the effects of tobacco are accounted [43]. Longterm use of inhaled cannabis has not been associated with increased risk of lung or gastrointestinal cancers [44], al- though a meta-analysis found evidence of premalignant changes in the respiratory tract [45]. There is some evidence that among individuals with pre-existing cardiac disease, cannabis users have an increased risk of myocardial infarction in the hour after smoking cannabis compared to nonusers [46]. A recent meta-analysis showed no major residual effects on neurocognitive functioning in long term dailyusers of cannabis [47]. THC rapidly crosses the placenta and accumulates in breast milk of nursing mothers [3], but there is no systematic evidence of direct or behavioral teratogenicity.

In reviewing the possible acute and long term adverse effects of cannabinoids as therapeutic agents one needs also to be mindful that other agents that are used for treatment of pain or spasticity also have adverse effects. Opioids produce sedation, nausea, constipation and dependence, withdrawal from which results in serious abstinence syndrome with much more severe effects - e.g. severe autonomic, gastrointestinal, and psychiatric - than the rather mild cannabis withdrawal phenomena. Tricyclic antidepressants and antiepileptic drugs commonly prescribed for chronic pain have psychotropic (e.g. sedation), anticholinergic (e.g. constipation, dizziness, palpitations, visual disturbance, urinary retention), and neuromuscular effects. Drugs for spasticity produce sedation (e.g. baclofen), hypotension (e.g. tizanidine) and serious interactions with antibiotics (e.g. tizanidine and ciprofloxacin). Benzodiazepines that are sometimes prescribed for spasticity can produce sedation, psychomotor incoordination, memory lapses, and paradoxical reactions, as well as dependence and withdrawal syndromes. Opioids and sedative-hypnotics are also drugs of abuse, and their ability to induce physiological dependence and serious withdrawal states exceed those of cannabis. Therefore, judgements on relative benefits and risks of cannabinoids as medicines need to be viewed within the broader context of risk-benefit of other agents as well [48].

\section{PATIENT SELECTION FOR CANNABINOID THER- APY}

Oral THC (eg, dronabinol) is FDA-approved as a second line agent for chemotherapy-associated nausea and emesis, and appetite stimulation. Dronabinol (and nabilone) have some evidence of efficacy for chronic neuropathic pain; whole plant cannabis extracts delivered by capsule or oralmucosal spray has been approved in Europe for analgesia in neuropathic pain and control of painful muscle spasticity. Patient selection for these agents would seem to be rather straightforward, and focus on therapeutic response to conventional treatments, consideration of possible psychotropic (eg, sedation effects if combined with alcohol) and cardiovascular effects, risk of dependence and an abstinence syndrome, and acknowledgement that there is narrow empirical basis for efficacy compared to standard treatments. Patients should be educated about expected adverse effects. The pharmacokinetics of orally administered cannabinoids would seem to decrease likelihood of diversion or abuse.

On the other hand, prescription of inhaled cannabis for medical purposes is legal in some US jurisdictions, and neurologic consultants, who are likely to be asked about the advisability of prescribing or recommending "medical mari- 
juana," may be justifiably uncertain of how to proceed. There are no published consensus statements or systematic approaches to identify candidates for "medical marijuana” or guide treatment; although some regulatory agencies, such as the Medical Board and the Office of the Attorney General of California have proposed guidelines (Table $\mathbf{1}$ ).

It should be noted that the evidence for efficacy is based primarily on relatively few short-term studies with small sample sizes of selected, mostly neuropathic pain conditions (ie, Phase II/III trials). "Medical cannabis", now available from dispensaries in some jurisdictions, is not subject to governmental standardization, and its constituents and potency are consequently unknown. Moreover, the mean potency of marijuana seized by federal and state authorities has more than doubled over the past 15 years to about 6\% THC, well over $20 \%$ of confiscated plants have a potency exceeding 9\%, and some specimens exceed 25\% THC [49]. Thus, cannabis obtained from dispensaries or other sources may have potency far exceeding that used in clinical trials described in this review. Furthermore, cannabis elicits concerns among regulators, clinicians, and patients regarding issues of misuse, abuse, and other liabilities. With these facts in mind, a potentially useful framework for evaluating advisability of medical marijuana are guidelines released by professional pain societies concerning prescription of long-term opioid therapy for chronic, non-cancer pain [50, 51]. The guidelines are framed by several questions. One question regards not only the legality of cannabis, but the standard of practice in the clinician's community, since either prescription or recommendation for use of cannabis is outside of "conventional practice.” As with prescription of opioids there are potential issues of legal liability [52]. A second question asks whether other treatments offer a more favorable risk-benefit ratio. The answer depends upon a careful differential diagnosis, identification of a potentially responsive pain syndrome (e.g., the strongest evidence is for neuropathic pain), consideration of other approaches (e.g., disease-modifying treatment, ablative interventions, other analgesics like anticonvulsants, noradrenergic antidepressants, opioids, or nonsteroidals, and cognitive-behavioral or rehabilitative therapy, or complementary treatments). A third question is whether there are medical and psychiatric risks. The shorter-term medical risks of cannabis are relatively low overall. Risks of hypotension and tachycardia should be evaluated in patients with cardiovascular diseases, as these may be associated with elevated risk of cannabis-associated myocardial infarction. Elderly patients with neurocognitive impairment may be predisposed to adverse effects of cannabis on memory and concentration, while even intact older individuals may be susceptible to over-sedation, and falls due to ataxia. The intoxicating effects of cannabis may be disturbing. A history of severe anxiety or paranoia on prior exposure to cannabis should be sought and would be a contraindication; since patients with serious mental illness (bipolar disorder or schizophrenia) may be particularly vulnerable to these adverse effects, they are unlikely to be candidates.

Moreover, there must be assessment of the potential for misuse, abuse, or addiction. This requires a careful examination for history of substance use disorders, and psychiatric illness, perhaps supplemented by formal psychiatric consultation. Screening questionnaires, such as the Screener and Opioid Assessment for Patients with Pain (SOAPP-R) [53], have been validated in chronic pain clinic populations to stratify patients into "lower" or "higher" risk of future opioid-related aberrant behaviors, and suitably modified might be applicable to assessment of risks for cannabis misuse. Most pain experts consider sobriety a foundation of successful pain treatment. Cannabis use is prevalent in chronic pain patients prescribed opioids and may be associated with current or future opioid misuse [54]. Patients screened "at risk" for aberrant opioid use, or a history of cannabis or other substance use disorders usually would not be considered eligible for medicinal cannabis. There might be exceptions. For example, there is some evidence that patients with sustained remission from alcohol dependence (ie, sober for $\geq 5$ years) are at no greater risk for developing a new onset substance abuse problem than non-alcohol controls, so this population would not necessarily be excluded automatically [55]. Also, a recent randomized trial suggests highlystructured approaches may result in successful analgesia and restoration of function without aberrant opioid use in "high risk" patients prescribed opioids for chronic pain [56]. Such methods, which involve a regimen of systematic urine toxicology testing, use of compliance checklists to evaluate for "red flags" of non-adherence to the program (eg, unsanctioned dose escalations, illicit drug use), and enrollment in a substance misuse counseling [56], might be adapted for use in high-risk candidates for medical marijuana. Before embarking on a trial of medical marijuana in patients with prior history of substance use disorders, it would be prudent to

Table 1. Medical Board/Office of the Attorney General of California Guidelines for Medical Marijuana

\begin{tabular}{|ll|}
\hline \multicolumn{1}{|c|}{ Physicians Recommending Medical Marijuana Need to: } \\
\hline \hline 1 & Take a history and conduct a good faith examination of the patient; \\
2 & Develop a treatment plan with objectives; \\
3 & Provide informed consent, including discussion of side effects; \\
4 & Periodically review the treatment's efficacy; \\
5 & Obtain consultations, as necessary; and \\
6 & Keep proper records supporting the decision to recommend the use of medical marijuana. \\
\hline http://www.mbc.ca.gov/board/media/releases_2004_05-13_marijuana.html \\
http://ag.ca.gov/cms_attachments/press/pdfs/n1601_medicalmarijuanaguidelines.pdf
\end{tabular}


establish a similar routine of urine toxicology testing, compliance checklists, and co-enrollment in a formal substance abuse treatment facility, just as is recommended for a trial of opioid analgesics [52]. Finally, chronic pain may be associated with major depression, which complicates treatment, and which must be diagnosed and independently treated for successful pain management. All of these factors being considered, if the decision is made to proceed, the clinician must formulate and document a treatment plan and the patient's agreement to abide by whatever guidelines are established. The clinical "trial" would entail establishing a therapeutic "dose," appropriately monitoring for adverse effects and misuse, and assessing outcome in terms of pain, mood, and function. Based on the literature of efficacy in neuropathic pain, there could be evidence of an effect within a minimum of two weeks. Response rates have been noted to increase, however, between two and four weeks in previous neuropathic pain registration trials for gabapentin and duloxetine [57]; one might select a longer duration in difficult-to-treat cases. Considering all these factors, one would then decide with the patient whether continued treatment is warranted. A possible algorithm to guide physician decision-making is presented in Fig. (1).

\section{CONCLUSION}

Evidence is accumulating that cannabinoids may be useful medicine for certain indications. Control of nausea and vomiting and the promotion of weight gain in chronic inanition are already licensed uses of oral THC (dronabinol cap-

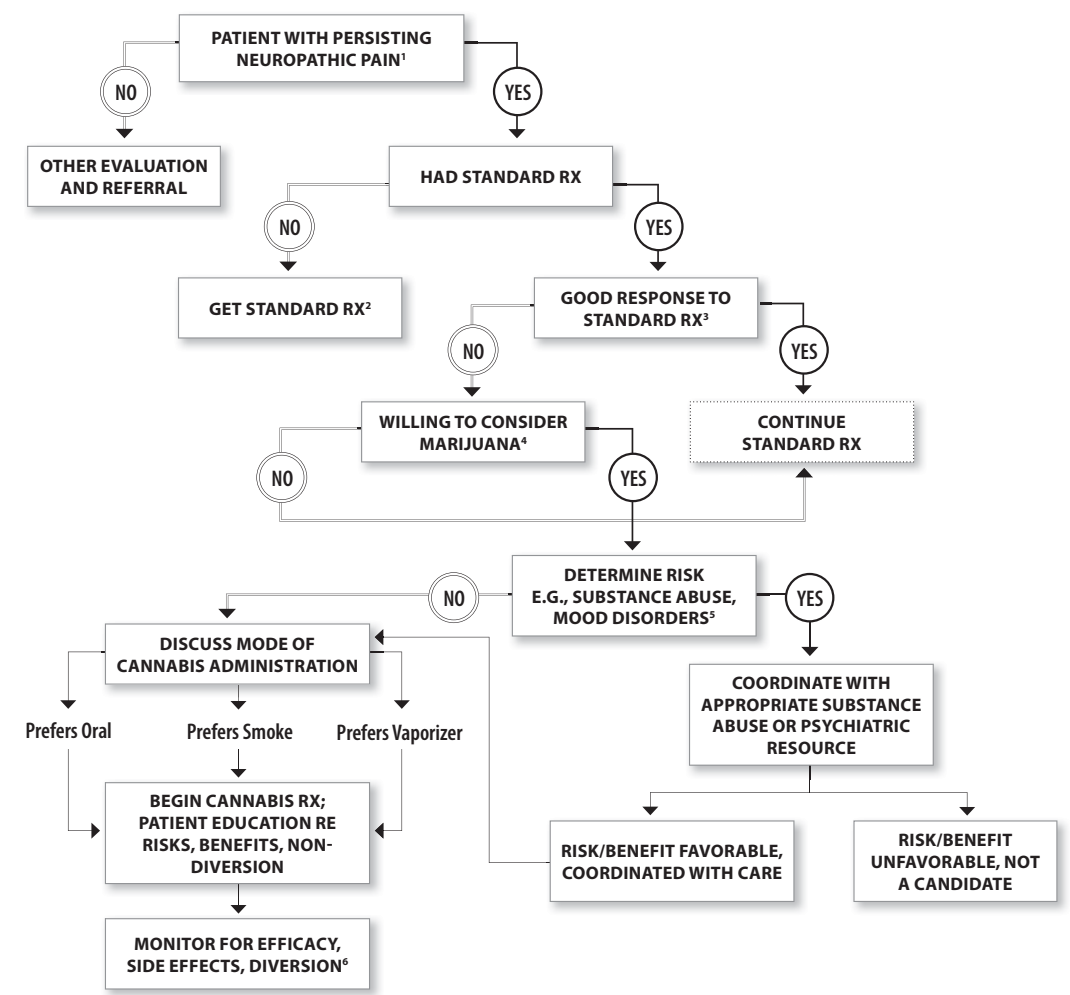

Fig. (1). A decision tree approach for physicians who may be considering recommending medicinal cannabis to a patient. This decision tree suggests some key points that a physician may need to consider in making his/her determination. In this case, a patient is assumed to present with persistent neuropathic pain. Initially, a determination needs to be made that the patient's signs and symptoms are indeed consistent with this diagnosis. Assuming a patient does not respond favorably to more standard treatments (e.g., antidepressants, anticonvulsants, etc), or cannot tolerate those, and the patient is willing to consider medicinal cannabis, the physician needs to determine risk versus benefit. Among these considerations is whether there is a history of substance abuse or serious psychiatric disorder that might be exacerbated by medicinal cannabis. Even if such risks exists, this does not necessarily preclude the use of medicinal cannabis; rather coordination with appropriate substance abuse and psychiatric resources is necessary, and based on such consultation a risk benefit ratio can be determined. In patients in whom the ratio appears favorable, the physician needs to discuss alternative modes of cannabis administration which may include oral, smoked, or vaporized systems. Once risks and benefits are evaluated and discussed with the patient, cannabis treatment may commence as with other psychotropic medications, with attention being paid to side effects as well as efficacy. In addition, there needs to be attentiveness to potential for misuse and diversion, which can then trigger a decision to discontinue.

\section{Key}

1. Daily or almost daily pain with typical neuropathic characteristics for at least 3 months; affects life quality.

Standard Rx = e.g., antidepressants, anticonvulsants; opioids; nonsteroidal anti-inflammatory drugs.

For example, at least $30 \%$ reduction in pain intensity.

Consider past experience, possible past history of side effects; willingness to smoke.

5. Determine history of substance abuse. If yes, or at "high risk" aberrant for drug behavior; proceed with close observation; possibly coordinate with substance abuse treatment program.

6. Efficacy $=$ at least $30 \%$ reduction in pain intensity. 
sules). Recent research indicates that cannabis may also be effective in the treatment of painful peripheral neuropathy and muscle spasticity from conditions such as multiple sclerosis [58]. Other indications have been proposed, but adequate clinical trials have not been conducted. As these therapeutic potentials are confirmed, it will be useful if marijuana and its constituents can be prescribed, dispensed, and regulated in a manner similar to other medications that have psychotropic effects and some abuse potential. Given that we do not know precisely which cannabinoids or in which combinations achieve the best results, larger and more representative clinical trials of the plant product are warranted. Because cannabinoids are variably and sometimes incompletely absorbed from the gut, and bioavailability is reduced by extensive first pass metabolism, such trials should include delivery systems that include smoking, vaporization, and oral mucosal spray in order to achieve predictable blood levels and appropriate titration. Advances in understanding the medical indications and limitations of cannabis in its various forms should facilitate the regulatory and legislative processes.

The classification of marijuana as a Schedule I drug as well as the continuing controversy as to whether or not cannabis is of medical value [59] are obstacles to medical progress in this area. Based on evidence currently available the Schedule I classification is not tenable; it is not accurate that cannabis has no medical value, or that information on safety is lacking. It is true cannabis has some abuse potential, but its profile more closely resembles drugs in Schedule III (where codeine and dronabinol are listed). The continuing conflict between scientific evidence and political ideology will hopefully be reconciled in a judicious manner [60, 61]. In the meantime, the decision to recommend this treatment in jurisdictions where use of medical marijuana is already permitted needs to be based on a careful assessment that includes proper diagnosis of a condition for which there is evidence that cannabis may be effective, along with consideration as to response to more standard treatments. Prior substance abuse history, psychiatric comorbidity, and other factors need to be weighed in a risk benefit analysis. Part of this analysis should consider that the potential longer-term harms of the cannabinoids are not fully understood: these include abuse and a dependence syndrome, adverse psychiatric and medical effects in vulnerable populations, and documented risk to traffic safety when combined with alcohol, and perhaps singly [62]. In the long term, as further studies demonstrate whether cannabis is effective for various indications, this should lead to development of novel modulators of the endocannabinoid system which may be prescribed and used as more traditional medicines.

\section{CONFLICTS OF INTEREST}

This work was supported by the University of California Center for Medicinal Cannabis Research. I. Grant served as consultant to Abbott Pharmaceuticals. J. H. Atkinson has served as a consultant to Eli Lilly and Company. B.L. Wilsey has served as a consultant to Ortho-McNeil Janssen Pharmaceuticals, Inc.

\section{ACKNOWLEDGEMENT}

Supported by the Center for Medicinal Cannabis Research at the University of California, San Diego.

\section{REFERENCES}

[1] Campbell FA, Tramer MR, Carroll D, Reynolds DJ, Moore RA, McQuay HJ. Are cannabinoids an effective and safe treatment option in the management of pain? A qualitative systematic review. BMJ 2001; 323(7303):13-6.

[2] Noyes R, Brunk SF, Avery DA, Canter AC. The analgesic properties of delta-9-tetrahydrocannabinol and codeine. Clin Pharmacol Ther 1975;18(1): 84-9.

[3] Huestis MA. Pharmacokinetics and metabolism of the plant cannabinoids, delta9-tetrahydrocannabinol, cannabidiol and cannabinol. Handb Exp Pharmacol 2005; (168): 657-90.

[4] Abrams DI, Jay CA, Shade SB, et al. Cannabis in painful HIVassociated sensory neuropathy: a randomized placebo-controlled trial. Neurology 2007; 68(7): 515-21.

[5] Ellis RJ, Toperoff W, Vaida F, et al. Smoked medicinal cannabis for neuropathic pain in HIV: a randomized, crossover clinical trial. Neuropsychopharmacol 2009; 34(3): 672-80.

[6] Wilsey B, Marcotte T, Tsodikov A, et al. A randomized, placebocontrolled, crossover trial of cannabis cigarettes in neuropathic pain. J Pain 2008; 9(6): 506-21.

[7] Farrar JT, Young JP Jr, LaMoreaux L, Werth JL, Poole RM. Clinical importance of changes in chronic pain intensity measured on an 11-point numerical pain rating scale. Pain 2001; 94(2):14958.

[8] Wallace M, Schulteis G, Atkinson JH, et al. Dose-dependent effects of smoked cannabis on capsaicin-induced pain and hyperalgesia in healthy volunteers. Anesthesiology 2007;107(5):785-96.

[9] Ware MA, Wang T, Shapiro S, et al. Smoked cannabis for chronic neuropathic pain: a randomized controlled trial. CMAJ 2010;182(14): E694-701.

[10] Zajicek J, Fox P, Sanders H, et al. Cannabinoids for treatment of spasticity and other symptoms related to multiple sclerosis (CAMS study): multicentre randomised placebo-controlled trial. Lancet 2003; 362(9395): 1517-26.

[11] Svendsen KB, Jensen TS, Bach FW. Does the cannabinoid dronabinol reduce central pain in multiple sclerosis? Randomised double blind placebo controlled crossover trial. BMJ 2004; 329(7460):253.

[12] Frank B, Serpell MG, Hughes J, Matthews JN, Kapur D. Comparison of analgesic effects and patient tolerability of nabilone and dihydrocodeine for chronic neuropathic pain: randomised, crossover, double blind study. BMJ 2008; 336(7637):199-201.

[13] Tramer MR, Carroll D, Campbell FA, Reynolds DJ, Moore RA, McQuay HJ. Cannabinoids for control of chemotherapy induced nausea and vomiting: quantitative systematic review. BMJ 2001; 323(7303):16-21.

[14] Ben Amar M. Cannabinoids in medicine: A review of their therapeutic potential. J Ethnopharmacol 2006; 105(1-2):1-25.

[15] Beal JE, Olson R, Laubenstein L, et al. Dronabinol as a treatment for anorexia associated with weight loss in patients with AIDS. J Pain Symptom Manag 1995;10(2): 89-97.

[16] Beal JE, Olson R, Lefkowitz L, et al. Long-term efficacy and safety of dronabinol for acquired immunodeficiency syndromeassociated anorexia. J Pain Symptom Manag 1997; 14(1):7-14.

[17] Pertwee RG. Cannabinoids and multiple sclerosis. Mol Neurobiol 2007; 36(1): 45-59.

[18] Wade DT, Robson P, House H, Makela P, Aram J. A preliminary controlled study to determine whether whole-plant cannabis extracts can improve intractable neurogenic symptoms. Clin Rehabil 2003;17(1): 21-9.

[19] Vaney C, Heinzel-Gutenbrunner M, Jobin P, et al. Efficacy, safety and tolerability of an orally administered cannabis extract in the treatment of spasticity in patients with multiple sclerosis: a randomized, double-blind, placebo-controlled, crossover study. Mult Scler 2004; 10(4): 417-24.

[20] Wade DT, Makela P, Robson P, House H, Bateman C. Do cannabis-based medicinal extracts have general or specific effects on symptoms in multiple sclerosis? A double-blind, randomized, 
placebo-controlled study on 160 patients. Mult Scler 2004; 10(4): 434-41.

[21] Abrams DI, Vizoso HP, Shade SB, Jay C, Kelly ME, Benowitz NL. Vaporization as a smokeless cannabis delivery system: a pilot study. Clin Pharmacol Ther 2007; 82(5): 572-8.

[22] Rog DJ, Nurmikko TJ, Friede T, Young CA. Randomized, controlled trial of cannabis-based medicine in central pain in multiple sclerosis. Neurology 2005; 65(6):812-9.

[23] Nurmikko TJ, Serpell MG, Hoggart B, Toomey PJ, Morlion BJ, Haines D. Sativex successfully treats neuropathic pain characterised by allodynia: a randomised, double-blind, placebocontrolled clinical trial. Pain 2007 ;133(1-3): 210-20.

[24] Johnson JR, Burnell-Nugent M, Lossignol D, Ganae-Motan ED, Potts R, Fallon MT. Multicenter, double-blind, randomized, placebo-controlled, parallel-group study of the efficacy, safety, and tolerability of THC:CBD extract and THC extract in patients with intractable cancer-related pain. J Pain Symptom Manag 2010; 39(2): 167-79.

[25] Collin C, Davies P, Mutiboko IK, Ratcliffe S. Randomized controlled trial of cannabis-based medicine in spasticity caused by multiple sclerosis. Eur J Neurol 2007; 14(3): 290-6.

[26] Wade DT, Collin C, Stott C, Duncombe P. Meta-analysis of the efficacy and safety of Sativex (nabiximols), on spasticity in people with multiple sclerosis. Mult Scler 2010; 16(6): 707-14.

[27] Wade DT, Makela PM, House H, Bateman C, Robson P. Longterm use of a cannabis-based medicine in the treatment of spasticity and other symptoms in multiple sclerosis. Mult Scler 2006; 12(5): 639-45.

[28] Farrar JT, Troxel AB, Stott C, Duncombe P, Jensen MP. Validity, reliability, and clinical importance of change in a 0-10 numeric rating scale measure of spasticity: a post hoc analysis of a randomized, double-blind, placebo-controlled trial. Clin Ther 2008; 30(5): 974-85.

[29] Jones RT, Benowitz NL, Herning RI. Clinical relevance of cannabis tolerance and dependence. J Clin Pharmacol 1981; 21(8-9 Suppl):143S-52S.

[30] Zajicek JP, Sanders HP, Wright DE, et al. Cannabinoids in multiple sclerosis (CAMS) study: safety and efficacy data for 12 months follow up. J Neurol Neurosurg Psychiatry 2005; 76(12):1664-9.

[31] Haney M, Ward AS, Comer SD, Foltin RW, Fischman MW. Abstinence symptoms following oral THC administration to humans. Psychopharmacology (Berl) 1999;141(4): 385-94.

[32] Haney M, Ward AS, Comer SD. Foltin RW, Fischman MW. Abstinence symptoms following smoked marijuana in humans. Psychopharmacology (Berl) 1999;141(4): 395-404.

[33] Budney AJ, Hughes JR, Moore BA, Vandrey R. Review of the validity and significance of cannabis withdrawal syndrome. Am J Psychiatry 2004; 161(11): 1967-77.

[34] Williamson EM. Drug interactions between herbal and prescription medicines. Drug Safety 2003; 26: 1075-92.

[35] Wills S. Drugs of Abuse. 2nd ed. London: Pharmaceutical Press 2005.

[36] Moore TH, Zammit S, Lingford-Hughes A, et al. Cannabis use and risk of psychotic or affective mental health outcomes: a systematic review. Lancet 2007; 370(9584): 319-28.

[37] D'Souza DC, Sewell RA, Ranganathan M. Cannabis and psychosis/schizophrenia: human studies. Eur Arch Psychiatry Clin Neurosci 2009; 259(7): 413-31.

[38] McGrath J, Welham J, Scott J, et al. Association between cannabis use and psychosis-related outcomes using sibling pair analysis in a cohort of young adults. Arch Gen Psychiatry 2010; 67(5): 440-7.

[39] van Winkel R. Family-based analysis of genetic variation underlying psychosis-inducing effects of cannabis: sibling analysis and proband follow-up. Arch Gen Psychiatry 2011; 68(2): 148-57.

[40] Miller LL, McFarland D, Cornett TL, Brightwell D. Marijuana and memory impairment: effect on free recall and recognition memory. Pharmacol Biochem Behav 1977; 7(2): 99-103.
[41] Sewell RA, Poling J, Sofuoglu M. The effect of cannabis compared with alcohol on driving. Am J Addict 2009; 18(3):185-93.

[42] Hall W, Degenhardt L. Adverse health effects of non-medical cannabis use. Lancet 2009;374(9698):1383-91.

[43] Tetrault JM, Crothers K, Moore BA, Mehra R, Concato J, Fiellin DA. Effects of marijuana smoking on pulmonary function and respiratory complications: a systematic review. Arch Intern Med 2007; 167(3): 221-8.

[44] Hashibe M, Morgenstern H, Cui Y, et al. Marijuana use and the risk of lung and upper aerodigestive tract cancers: results of a population-based case-control study. Cancer Epidemiol Biomark Prev 2006;15(10):1829-34.

[45] Mehra R, Moore BA, Crothers K, Tetrault J, Fiellin DA. The association between marijuana smoking and lung cancer: a systematic review. Arch Intern Med 2006 ;166(13):1359-67.

[46] Mittleman MA, Lewis RA, Maclure M, Sherwood JB, Muller JE. Triggering myocardial infarction by marijuana. Circulation 2001; 103(23): 2805-9.

[47] Grant I, Gonzalez R, Carey CL, Natarajan L, Wolfson T. Nonacute (residual) neurocognitive effects of cannabis use: a metaanalytic study. J Int Neuropsychol Soc 2003; 9(5):679-89.

[48] Brunton L, Chabner B, Knollman B, eds. Goodman \& Gilman's The Pharmacological Basis of Therapeutics. 12th ed. New York, NY: McGraw-Hill 2010.

[49] Mehmedic Z, Chandra S, Slade D, et al. Potency trends of Delta9THC and other cannabinoids in confiscated cannabis preparations from 1993 to 2008. J Forensic Sci 2010; 55(5): 1209-17.

[50] Chou R, Fanciullo GJ, Fine PG, et al. Clinical guidelines for the use of chronic opioid therapy in chronic noncancer pain. J Pain 2009; 10(2): 113-30.

[51] Fine PG, Portenoy RK. A Clinical Guide to Opioid Analgesia. 2nd ed. New York: Vendome Group 2007.

[52] Jamison RN, Clark JD. Opioid medication management: clinician beware! Anesthesiology 2010; 112(4):777-8.

[53] Butler SF, Fernandez K, Benoit C, Budman SH, Jamison RN. Validation of the revised screener and opioid assessment for Patients with Pain (SOAPP-R). J Pain 2008; 9(4): 360-72.

[54] Reisfield GM, Wasan AD, Jamison RN. The prevalence and significance of cannabis use in patients prescribed chronic opioid therapy: a review of the extant literature. Pain Med 2009; 10(8): 1434-41.

[55] Schuckit MA. Drug and alcohol abuse: a clinical guide to diagnosis and treatment. 6th ed. New York: Springer 2006.

[56] Jamison RN, Ross EL, Michna E, Chen LQ, Holcomb C, Wasan AD. Substance misuse treatment for high-risk chronic pain patients on opioid therapy: a randomized trial. Pain 2010;150(3):390-400.

[57] Ho TW, Backonja M, Ma J, Leibensperger H, Froman S, Polydefkis M. Efficient assessment of neuropathic pain drugs in patients with small fiber sensory neuropathies. Pain 2009;141(12):19-24.

[58] Corey-Bloom J, Wolfson T, Gamst A, Jin S, Marcotte TD, Bentley $\mathrm{H}$, Gouaux B. A randomized, placebo-controlled trial of smoked cannabis for spasticity in multiple sclerosis. CMAJ (In press).

[59] Drug Enforcement Agency. The DEA Position on Marijuana. January 2011; Available from: http://www.justice.gov/dea/marijuana_position.pdf. [cited 2012 March 26]

[60] Cohen PJ. Medical marijuana: the conflict between scientific evidence and political ideology. Part two of two. J Pain Palliat Care Pharmacother 2009; 23(2): 120-40.

[61] Cohen PJ. Medical marijuana: the conflict between scientific evidence and political ideology. Part one of two. J Pain Palliat Care Pharmacother 2009; 23(1): 4-25.

[62] Asbridge M, Hayden JA, Cartwright JL. Acute cannabis consumption and motor vehicle collision risk: systematic review of observational studies and meta-analysis. BMJ 2012; 344:e536

(C) Igor Grant; Licensee Bentham Open.

This is an open access article licensed under the terms of the Creative Commons Attribution Non-Commercial License (http://creativecommons.org/licenses/by-nc/3.0/) which permits unrestricted, non-commercial use, distribution and reproduction in any medium, provided the work is properly cited. 